\title{
The Use of Bovine Collagen and Skin Graft for Reconstruction of the Glans Penis Following Cancer Resection
}

\author{
Zhongyi Sun, Varsha R. Sinha, Ezekiel E. Young, Brian Bensadigh, Carmen Ceron, Bruce R. Kava, \\ Jennifer N. Landon, Christopher J. Salgado* \\ Division of Plastic Surgery, DeWitt Daughtry Department of Surgery, Leonard M. Miller School of Medicine, \\ University of Miami, Miami, USA \\ Email: * christophersalgado@med.miami.edu
}

Received August 21, 2012; revised September 27, 2012; accepted October 4, 2012

\begin{abstract}
Penile reconstruction following cancer resection has long been a challenge due to demand for both aesthetic and functional preservation. Although various methods have attempted to achieve both goals, none have succeeded entirely. In this report, we introduce a novel reconstructive technique on a 64-year-old patient with squamous cell carcinoma of the glans penis. The technique consisted of two staged procedures. First, a dermal substitute, consisting of bovine collagen, was sutured onto the defect after partial glansectomy and three weeks later, the integrated dermis was covered with autologous non-meshed partial thickness skin graft. Use of this dermal substitute in a staged fashion allowed for ability to await final pathologic margins prior to definitive reconstruction and increased dermal thickness. After 9-month follow-up, sexual function returned within 3 months and the patient remained healed. We found this technique to be useful in concurrently preserving penile function and cosmesis. Future studies should include more patients and longer follow-up.
\end{abstract}

Keywords: Glans Penis; Bovine Collagen; Integra; Penis

\section{Introduction}

In recent years there has been increasing emphasis placed on maintenance of appearance and function in the surgical management of penile cancers. This shift has involved efforts to both limit the amount of non-malignant tissue removed, and to reconstruct the penis as accurately as possible after resection. Since the vast majority of penile cancers occur on the glans or prepuce, many different glanuloplasty techniques have been proposed. Unfortunately, it has proven quite difficult to reconstruct the glans in such a manner as to provide natural appearance, and effective voiding capacity, sensation, and erectile function. None of the techniques described thus far have met all these demands, yielding fully satisfactory outcomes. Here we describe a novel technique using Integra (Integra Life Sciences, Plainsboro, NJ, USA), a bilayer matrix wound dressing. This method was used in a patient with squamous cell carcinoma in situ limited to the glans. After nine months of follow-up, it appears to provide excellent cosmesis and preservation of function.

\section{Case Report}

A 64-year-old, otherwise healthy, male presented with an

*Corresponding author. ulcerated lesion on his glans penis. Physical exam revealed a $3 \times 5 \times 2 \mathrm{~cm}$ verrucous plaque encompassing a large portion of the glans surface, extending from near the coronal sulcus out to the external urethral meatus (Figure 1). Prior punch biopsy had demonstrated severely atypical epithelial proliferation consistent with squamous cell carcinoma in situ. After partial glansectomy was performed, a $2 \times 2 \mathrm{~cm}$ sheet of Integra was placed over the defect. In addition to several quilting sutures, the Integra was also secured with sutures around the meatus, and circumferentially to the proximal skin edge (Figure 2). The foley catheter was removed after one week. Three weeks after the first procedure, the patient underwent removal of the Integra silicone layer and replacement with a non-meshed partial thickness skin graft. The graft was taken from excess right groin tissue, which was harvested during a combination inguinal hernia repair.

The patient recovered well and there were no complications. Within three months he returned to full sexual function. After nine months of follow-up he has continued to void well without any evidence of meatal stenosis. The graft has healed well with a fairly natural appearance (Figure 3). The patient reports complete satisfaction with both the function and appearance. 


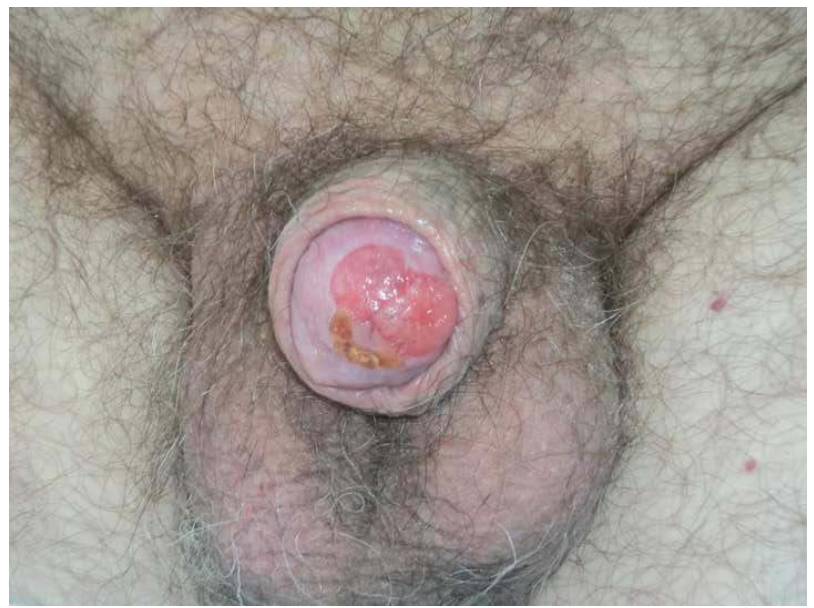

Figure 1. Preoperative photo demonstrating the lesion.

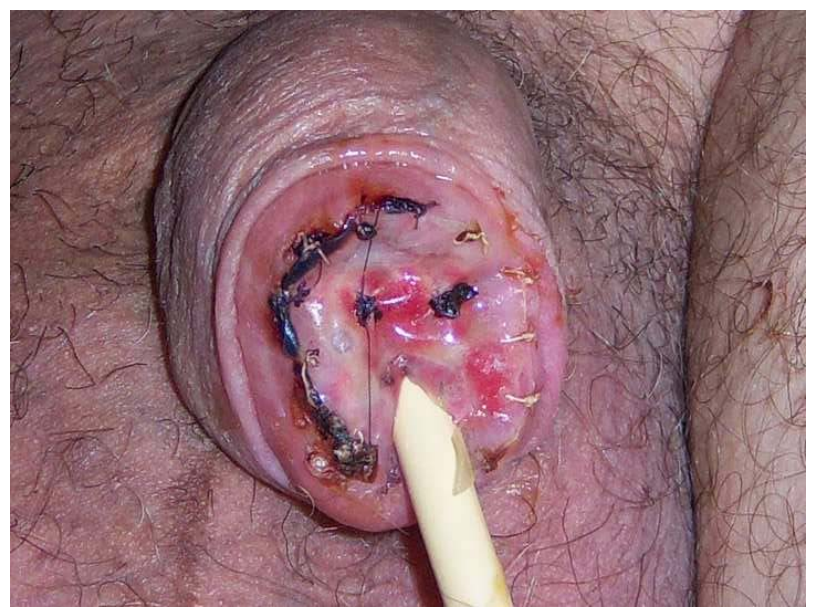

Figure 2. Photo taken immediately after the first stage procedure.

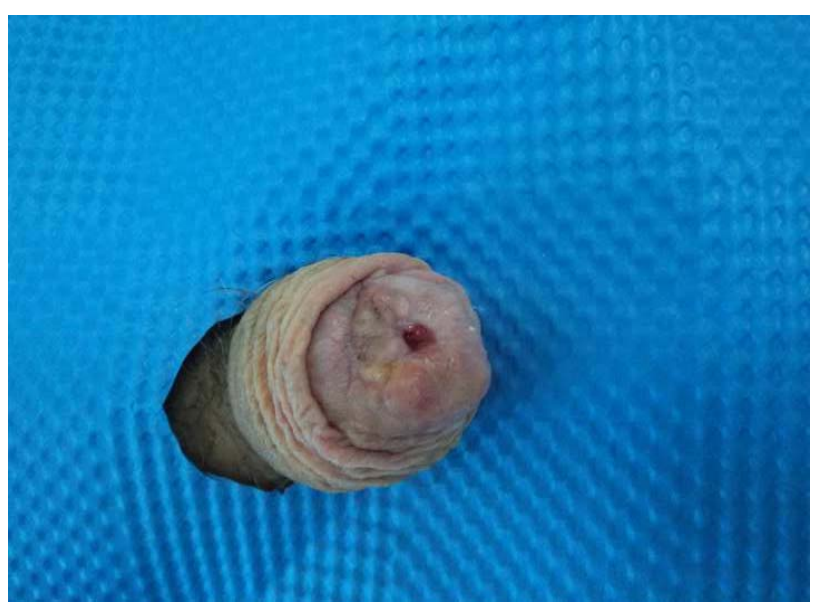

Figure 3. Photo six months after the second stage procedure.

\section{Discussion}

While many glans reconstruction techniques have been described each has significant shortcomings. Until re- cently, all these methods involved primary coverage of the defect with autologous tissue. Although Integra and other biologic skin substitutes have been utilized since 1997 in others areas of the body [1], the first penile application was for traumatic degloving avulsion injury described by Popescu in 2007 [2]. In 2009 there were two additional reports describing the use of Integra to cover the penile shaft, one after debridement for necrotizing fasciitis [3], and one after third-degree burns [4].

In 2010 Knotts published the first, and until now, only description of the use of biologic skin substitute for penile coverage after cancer resection [5]. This was also the only previous description of the use of such material on the glans penis. This manuscript described a patient similar to ours, with the exceptions that he had no urethral involvement, and had undergone a Moh's procedure resulting in significant volume deficiency. Because he was attempting to fill a cavitary defect, Knotts used the Integra in a very different manner than that described here. Instead of lying the material on top of the defect as a sheet, as is typically done, he removed the silicone layer from the start and "mulched" the material into a "putty consistency", that was then "sculpted to create the glans," and onto which he then applied unmodified Integra. In the second procedure Knotts' description also differs from ours in that he placed full-thickness, non-meshed skin grafts onto the Integra.

While Knotts' method appears to be useful in cases where the resection yields notable volume deficiency, the majority of cases do not necessitate this type of "mulch". Herein we offer the first description of the use of Integra in the traditional manner on the glans penis. This is also the first report describing Integra used in the traditional manner for skin coverage on any part of the penis after cancer resection, and the first reported case where Integra was used to fill a defect with urethral involvement.

\section{Conclusion}

Although, as of now, we can only report short-term results in a single patient, it appears that this dermal substitute can be utilized in this manner with excellent cosmesis and functional results. This method allows for simplification of wound care, avoiding pain from dressing changes. It also provides increased dermal thickness, which is particularly important in the glans, and allows for a delay in definitive reconstruction while final pathology is pending. Studies including more patients and longer follow-up are indicated.

\section{REFERENCES}

[1] C. Lorenz, A. Petracic, H. P. Hohl, et al., "Early Wound Closure and Early Reconstruction. Experience with a 
Dermal Substitute in a Child with 60 Percent Surface Area Burn," Burns, Vol. 23, No. 6, 1997, pp. 505-508. doi:10.1016/S0305-4179(97)00022-3

[2] S. Popescu, N. Ghetu, O. Grosu, et al., "Integra: A Therapeutic Alternative in Reconstructive Surgery. Our First Experience," Chirurgia, Vol. 102, No. 2, 2007, pp. 197204.

[3] C. E. Payne, A. M. Williams and N. B. Hart, "Lotus Petal Flaps for Scrotal Reconstruction Combined with Integra Resurfacing of the Penis and Anterior Abdominal Wall Following Necrotizing Fasciitis," Journal of Plastic, Re- constructive \& Aesthetic Surgery, Vol. 62, No. 3, 2009 , pp. 393-397.

[4] A. D. Jaskille, J. W. Shupp, J. C. Jeng and M. H. Jordan, "Use of Integra in the Treatment of Third Degree Burns to the Penile Shaft: A Case Series with 6-Month FollowUp," Journal of Burn Care \& Research, Vol. 30, 2009, pp. 524-528. doi:10.1097/BCR.0b013e3181a28d4b

[5] C. D. Knotts, A. L. Morgan and J. C. Deschamps-Braly, et al., "Mulching' Integra for Glans Penis," Plastic and Reconstructive Surgery, Vol. 126, No. 3, 2010, pp. 151e152e. doi:10.1097/PRS.0b013e3181e3b599 